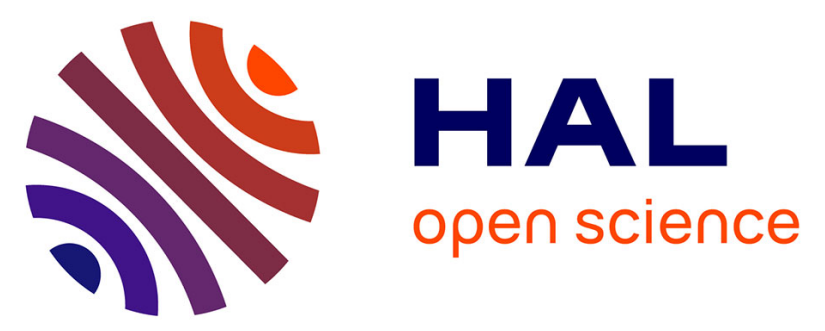

\title{
Ionization of water by (20-150)-keV protons: Separation of direct-ionization and electron-capture processes
}

\author{
F. Gobet, S. Eden, B. Coupier, J. Tabet, B. Farizon, M. Farizon, M.J.
}

Gaillard, M. Carré, S. Ouaskit, T.D. Märk, et al.

\section{- To cite this version:}

F. Gobet, S. Eden, B. Coupier, J. Tabet, B. Farizon, et al.. Ionization of water by (20-150)-keV protons: Separation of direct-ionization and electron-capture processes. Physical Review A : Atomic, molecular, and optical physics [1990-2015], 2004, 70, pp.062716-1-8. 10.1103/PhysRevA.70.062716 . in2p3-00023515

\author{
HAL Id: in2p3-00023515 \\ https://hal.in2p3.fr/in2p3-00023515
}

Submitted on 24 May 2021

HAL is a multi-disciplinary open access archive for the deposit and dissemination of scientific research documents, whether they are published or not. The documents may come from teaching and research institutions in France or abroad, or from public or private research centers.
L'archive ouverte pluridisciplinaire HAL, est destinée au dépôt et à la diffusion de documents scientifiques de niveau recherche, publiés ou non, émanant des établissements d'enseignement et de recherche français ou étrangers, des laboratoires publics ou privés. 


\title{
Ionization of water by (20-150)-keV protons: Separation of direct-ionization and electron-capture processes
}

\author{
F. Gobet, ${ }^{1}$ S. Eden, ${ }^{1}$ B. Coupier, ${ }^{1}$ J. Tabet,${ }^{1}$ B. Farizon, ${ }^{1}$ M. Farizon, ${ }^{1}$ M. J. Gaillard, ${ }^{1}$ M. Carré,${ }^{1,2}$ S. Ouaskit, ${ }^{1,3}$ \\ T. D. Märk, ${ }^{1,4}$ and P. Scheier ${ }^{1,4}$ \\ ${ }^{1}$ Institut de Physique Nucléaire de Lyon, IN2P3-CNRS et Université Claude Bernard Lyon 1, 43 boulevard du 11 Novembre 1918, \\ F-69622 Villeurbanne Cedex, France \\ ${ }^{2}$ Laboratoire de Spectrométrie Ionique et Moléculaire, CNRS et Université Claude Bernard Lyon 1, 43 boulevard du 11 Novembre 1918, \\ F-69622 Villeurbanne Cedex, France \\ ${ }^{3}$ Université Hassan II, Faculté des Sciences II, B.P. 6621-sidi Othermane, Casablanca, Morocco \\ ${ }^{4}$ Institut für Ionenphysik, Leopold Franzens Universität, Technikerstraße 25, A-6020 Innsbruck, Austria \\ (Received 16 December 2002; revised manuscript received 6 July 2004; published 20 December 2004)
}

\begin{abstract}
Mass analyzed product ions have been detected in coincidence with the projectile following the ionization of water by proton impact. Measurement of the projectile charge state postcollision enables the different ionization processes to be identified: direct ionization, single electron capture, and double electron capture. A complete set of partial and total absolute cross sections is reported for the direct ionization and electron capture processes initiated by proton collisions at $20-150 \mathrm{keV}$. The cross sections for the direct ionization of $\mathrm{H}_{2} \mathrm{O}$ by proton impact are compared with previous electron impact results [Straub et al., J. Chem. Phys. 108, 109 (1998)].
\end{abstract}

DOI: 10.1103/PhysRevA.70.062716

PACS number(s): $34.70 .+\mathrm{e}$

\section{INTRODUCTION}

The interactions between water molecules and projectiles such as photons, metastable atoms, electrons, and ions are of fundamental importance not only in Physics and Astronomy [1-4] but also in Biology and Medicine [5,6]. Incident rays can damage living tissue both as a result of direct particlebiomolecule interactions and through processes initiated by secondary species such as radicals formed by the dissociation of neighboring water molecules. Sanche and co-workers [7] recently demonstrated that detailed knowledge of the ionization processes is necessary to achieve a full understanding of biological radiation damage on a microscopic scale.

Wide-ranging interest in water has led to a number of recent cross section measurements for processes initiated by electron [8], positron [9] and ion impact [10,11]. Most work has featured the electron impact ionization of water $([8,12]$ and references therein) whereas ion impact experiments are very rare. Koopman [13] published total cross sections in 1968 for electron capture by $0.1-1.4 \mathrm{keV} \mathrm{He}{ }^{+}$in water vapor. In the same year, Toburen et al. [14] measured the total electron capture cross section for proton-water molecule collisions over the energy range 100 to $2500 \mathrm{keV}$. Doubly differential ionization cross sections for $300-1500 \mathrm{keV}$ proton impact on $\mathrm{H}_{2} \mathrm{O}$ were reported by Toburen and Wilson [15] in 1977. The same group measured doubly differential cross sections for electron ejection from $\mathrm{H}_{2} \mathrm{O}$ upon $\mathrm{He}^{+}$and $\mathrm{He}^{2+}$ impact between 300 and $2000 \mathrm{keV}$ [16]. Rudd et al. published two papers on the ionization and electron capture cross sections for 7-4000 keV proton [17] and 5-450 keV $\mathrm{He}^{+}$[18] collisions with water molecules. In 1986, Bolorizadeh and Rudd reported differential ionization cross sections for $\mathrm{H}_{2} \mathrm{O}$ upon electron [19], proton [20], and neutral hydrogen atom impact [21].

More recently, Werner et al. [10] used a position- and time-sensitive multiparticle detector to study the fragmenta- tion and multiple ionization of water following collisions with fast protons and $\mathrm{He}^{+}$ions. The first complete ion-impact induced molecular breakup pattern for more than two fragments was reported. The data provide information about total and partial single ionization, multiple ionization, fragmentation, kinetic energy release, and angular correlation for each individual impact event. However, Werner et al. [10] could not distinguish between direct ionization and electron capture processes.

In 2001, Gobet et al. [11] applied a novel event by event analysis technique to the proton impact ionization of water and detected product ions in coincidence with the outgoing projectile. The coincident charge state measurement of the projectile postcollision and of the product ions enabled the partial electron capture cross sections for ion impact to be determined the first time. The present paper provides a detailed description of the calibration techniques applied in order to obtain absolute cross sections. In contrast to the previous work [11] in which only the partial cross sections corresponding to $\mathrm{H}^{+}$and $\mathrm{H}_{2} \mathrm{O}^{+}$were reported, a full set of partial cross sections is presented over the incident energy range. This allows a detailed comparison to be made between the present proton impact data and the partial electron impact ionization cross sections of Straub et al. [8].

\section{EXPERIMENT}

The experiments were carried out using a newly developed crossed-beam apparatus shown schematically in Fig. 1. Pure molecular hydrogen is ionized in a standard RFdischarge source $(80 \mathrm{MHz})$. Typical parameters for the ion source are $30 \mathrm{~W}$ RF-power and a $\mathrm{H}_{2}$ pressure of $10^{-3}$ Torr. Beams of singly charged ions are accelerated to energies between 20 and $150 \mathrm{keV}$ with a resolution $\Delta E / E$ of 0.01 . The accelerator system is described in detail by Carré et al. 


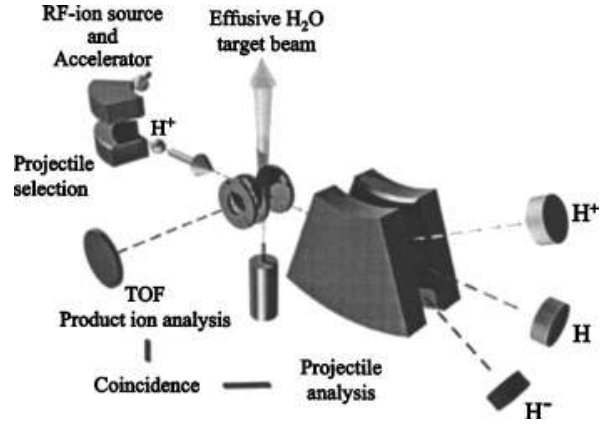

FIG. 1. Schematic diagram of the experimental system.

[22]. A magnetic sector field is used to separate protons from other ions such as $\mathrm{H}_{2}{ }^{+}, \mathrm{H}_{3}{ }^{+}$, and ions originating from impurities in the source. After collimation by means of two circular apertures of $0.5 \mathrm{~mm}$ radius set $1 \mathrm{~m}$ apart, the proton beam is crossed at right angles with an effusive beam of water molecules. Distilled water which has been degassed prior to the experiment through a series freezing, pumping and thawing cycles is kept at a temperature of $255 \mathrm{~K}$ throughout the measurements. The vapor above the ice is introduced through a capillary kept at room temperature. The charge state of the projectile after a collision with a water molecule is determined using a magnetic analyzer and three channeltrons located at the appropriate positions to detect $\mathrm{H}^{+}, \mathrm{H}^{0}$, and $\mathrm{H}^{-}$, respectively.

A home-built linear time-of-flight (TOF) mass spectrometer is used to investigate all the product ions formed by the impact of a proton upon a water molecule. The instrument is composed of an extraction region defined by parallel plates $( \pm 150 \mathrm{~V}, 1 \mathrm{~cm}$ apart $)$ on either side of the water beam, an acceleration region, a time-of-flight tube of length $120 \mathrm{~cm}$, and a channeltron detector. The product ions are extracted from the interaction region perpendicularly to both the proton and water beams and the system can be configured to detect either positive or negative ions. The extraction and acceleration fields are selected in fulfillment of the conditions defined by Wiley and McLaren [23] in order to focus incoming ions precisely at the detector entrance. The mass resolution of the TOF is sufficiently high to separate $\mathrm{H}_{2} \mathrm{O}^{+}, \mathrm{OH}^{+}$, and $\mathrm{O}^{+}$(see Fig. 2).

It is of great importance that the projectile beam does not contain fast hydrogen atoms formed by the neutralization of protons in collisions with the surfaces or the residual gas. Thus the background vacuum is maintained below $10^{-6}$ torr and the alignment of the proton beam is verified prior to each experiment. Furthermore, single collision conditions are necessary to guarantee the unambiguous identification of reactions. This is checked by adjusting the density of the water beam such that the total product ion yield varies over a factor range of 4 . The cross sections and branching ratios (the partial cross section corresponding to a specific product ion divided by the total ionization cross section) are observed to be independent of the changing target density to within $\pm 1 \%$. Therefore, it can be concluded that the product ion signal is uncontaminated by reactions between hydrogen atoms (neutralized by electron capture from a water molecule) and water molecules.

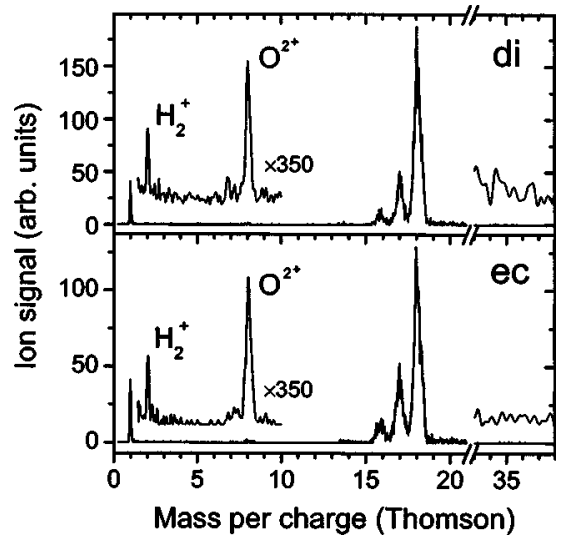

FIG. 2. Product ion mass spectrum for the ionization of $\mathrm{H}_{2} \mathrm{O}$ by proton impact at an energy of $50 \mathrm{keV}$. Upper diagram: ions produced by direct ionization (di) $\mathrm{H}^{+}+\mathrm{H}_{2} \mathrm{O} \rightarrow$ ions $+\mathrm{H}^{+}$. Lower diagram: ions produced by single electron capture $(\mathrm{ec}) \mathrm{H}^{+}+\mathrm{H}_{2} \mathrm{O}$ $\rightarrow$ ions + H. Magnified plots $(\times 350)$ : spectra averaged over the full range of incident energies studied (20-150 keV).

The determination of the mass-to-charge ratio of the product ions depends upon the detection of the corresponding projectile. Each proton that crosses the interaction region can be detected independently of its postinteraction charge state. The energy transfer during a collision with a water molecule is negligible in comparison with the kinetic energy of the proton beam (20-150 keV). Thus, the precise time at which the proton-water beam interaction takes place can be determined for each detected projectile. The arrival of a product ion produces a pulse at the channeltron detector of the TOF. The time difference between this pulse and the proton-water beam interaction equals the flight time of the product ion. Clearly, the number of projectiles has to be sufficiently low for each product ion signal to be correlated to exactly one projectile. Therefore, only one proton is allowed to cross the interaction region during a time interval equal to twice the flight time of the heaviest conceivable product ion. For the present experimental arrangement, this limits the primary ion beam current to 2000 protons per second.

In contrast to earlier experiments $[10,13-18,20]$, the present method simultaneously provides the mass-per-charge ratio of the product ions and an analysis of the projectile postinteraction. This enables direct ionization to be distinguished from electron capture during the ionization processes for each collision event. Furthermore, processes in which two or more product ions are formed in a single collision event can be identified. For example, the Coulomb explosion of multiply charged $\mathrm{H}_{2} \mathrm{O}$ molecules observed and studied in detail by Werner et al. [10] can also be identified in the present experiment.

\section{RESULTS AND DISCUSSION}

Figure 2 shows the sum of the product ion mass spectra corresponding to $50 \mathrm{keV}$ proton- $\mathrm{H}_{2} \mathrm{O}$ collisions. The data plotted in the upper part of the figure correspond to protons 
hitting the projectile detector (direct ionization) while the lower mass spectrum corresponds to the detection of neutralized projectiles (electron capture). Double electron capture [24], indicated by the detection of an $\mathrm{H}^{-}$projectile, is not observed in coincidence with product ions. In order to improve the statistics and reduce the noise for low intensity products, both graphs contain a second mass spectrum that was determined by averaging the ion signal for measurements over the full range of proton energies. This averaged ion signal is multiplied by 350 and plotted in Fig. 2 over the ranges 1.3-10 and 33-38 Thomson (mass number/ion charge). The absence of a peak at a mass per charge ratio of 36 Thomson indicates that the density of neutral clusters in the water beam must be negligible. Both averaged mass spectra reveal a clear peak at a mass per charge ratio of 2 Thomson. This represents the first observation of $\mathrm{H}_{2}{ }^{+}$formation by proton impact on $\mathrm{H}_{2} \mathrm{O}$, although the yield of this fragment ion is too small to determine its cross section at different projectile energies. Direct ionization and single electron capture contribute roughly equally to total ion production in $50 \mathrm{keV}$ collisions. However, the relative abundance of $\mathrm{H}_{2} \mathrm{O}^{+}$and fragment ions differs significantly for the two processes. Electron capture is observed to lead to larger fragment ion signals than direct ionization.

Substantial kinetic energy release in a dissociation process can complicate the analysis of fragment ions [25]. Low mass fragment ions, thus released at high velocity (momentum conservation), may be channeled to a detector by an extraction voltage with a lower efficiency than heavier fragments [26]. Due to the relatively small geometric acceptance for ions of the present time-of-flight assembly, it is essential to check the variation in detection efficiency for the various fragments. This is done by comparison with the absolute partial cross sections for the ionization of water by proton impact at $100-350 \mathrm{keV}$ obtained by Werner et al. [10] independently of any discrimination effects. Werner et al. only detected collision events producing at least one free electron. These events correspond to direct ionization or electron capture processes which lead to the formation of more than one ion or to a multiply charged product. Therefore, the present partial cross sections for double ionization and multi-ion formation by electron capture and for direct ionization are summed to make comparisons with the cross sections published by Werner et al. [10].

The detection of $\mathrm{H}_{2} \mathrm{O}^{+}$can be considered to be free of discrimination losses as there is no associated fragmentation process. The ratio of the fragment ion yield to the $\mathrm{H}_{2} \mathrm{O}^{+}$ion signal is independent of the target thickness and projectile ion beam properties but still contains information about the discrimination due to the kinetic energy of the fragment ion. In Fig. 3, these ion yield ratios are plotted as a function of the projectile energy (filled symbols). The figure also shows the cross sections for the production of fragment ions divided by the cross section for $\mathrm{H}_{2} \mathrm{O}^{+}$production published by Werner et al. [10] (open symbols). For the heavier singly charged fragment ions $\left(\mathrm{OH}^{+}\right.$and $\left.\mathrm{O}^{+}\right)$, the two data sets differ by less than $10 \%$. However, in the case of $\mathrm{H}+$ dissociation (up triangles), the present data are significantly lower than those of Werner et al. [10]. This confirms that the present TOF has a reduced detection efficiency for lighter fragment

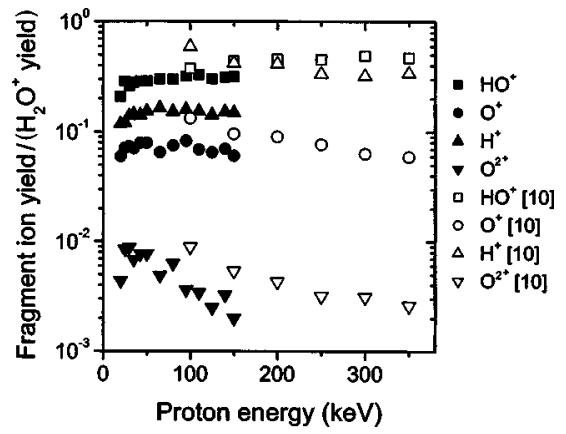

FIG. 3. Fragment ion yield divided by $\mathrm{H}_{2} \mathrm{O}^{+}$yield plotted against proton-water collision energy considering only those events where at least one free electron is produced (see text for details).

ions. For each fragment ion a factor was determined to correct for discrimination. These correction factors are given in Table I. The fragment ion yields presented in the remainder of this paper have been corrected accordingly. Thus it is assumed that the discrimination due to kinetic energy release is independent of incident proton energy within the range of the present experiments.

The present data are normalized to the absolute total cross sections for the ionization of water determined by Rudd et al. [17] for proton energies between 7 and $4000 \mathrm{keV}$. Rudd et al. also measured the cross sections $\left(\sigma_{-}\right)$for processes leading to the production of a negatively charged particle. The total cross section for the formation of a positively charged product ion was defined as $\sigma_{+}$. It was assumed that $\sigma_{-}$is exclusively due to the production of electrons and that anion formation can be neglected. The present experimental system allows the production of anions to be analyzed. In accordance with Rudd et al. [17], negatively charged ion products are not observed upon proton impact ionization of water. As carried out in the previous work [11], it is possible to calibrate the present measurements for total ion production using the $\sigma_{+}$data of Rudd et al. [17]. Furthermore, the part of the ion signal used to determine the correction factors related to the kinetic energy of fragment ions can be calibrated using $\sigma_{-}$in order to determine absolute cross sections. Except in the case of very low projectile energies, the two techniques lead to the same result.

Rudd et al. [17] proposed a formula for $\sigma_{-}$as a function of the proton energy $E_{p}$ :

TABLE I. Correction factors derived by comparing the present measurements of fragment ion yield over $\mathrm{H}_{2} \mathrm{O}^{+}$yield with the corresponding ratio from the data of Werner et al. [10]. Figure 3 shows these ratios as a function of the proton energy.

\begin{tabular}{cc}
\hline \hline Fragment ion & Correction factor \\
\hline $\mathrm{OH}^{+}$ & 1.29 \\
$\mathrm{O}^{+}$ & 1.68 \\
$\mathrm{O}^{2+}$ & 1.91 \\
$\mathrm{H}^{+}$ & 3.07 \\
\hline
\end{tabular}




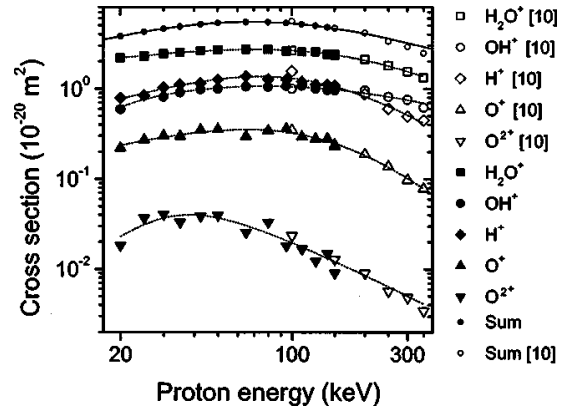

FIG. 4. Total and partial absolute ionization cross sections of $\mathrm{H}_{2} \mathrm{O}$ by proton impact considering only those events where at least one electron is produced (see text for details). The continuous and dashed lines correspond to total and partial cross sections, respectively, and are fitted to Eq. (1) formulated by Rudd et al. [17]. The corresponding parameters are given in Table II.

$$
\sigma_{-}\left(E_{p}\right)=\frac{4 \pi a_{0}^{2}}{\frac{x}{A \ln (1+x)+B}+\frac{1}{C x^{D}}},
$$

where $E_{p}$ is the proton energy in $\mathrm{eV}, x=\left(E_{p} / 1836\right) / 13.6$, and $a_{0}$ is the Bohr radius. The four constants, $A=2.89, B$ $=4.42, C=1.48$, and $D=0.75$, were determined by fitting Eq. (1) to the experimental total cross sections [17]. The sum of the present yield measurements (corrected for discrimination due to fragment kinetic energy) of all the different product ions for processes that release at least one electron is normalized to the value of Eq. (1). This calibration procedure provides absolute total and partial cross section curves for the various product ions in the energy range from 20 to $150 \mathrm{keV}$.

Figure 4 shows the present absolute cross sections obtained using the calibration described above (filled symbols) in comparison with the data of Werner et al. [10] (open symbols). The curves represent fits to Eq. (1) for both data sets (see Table II for the corresponding constants). The present values represent a smooth extension of the data of Werner et al. [10] to lower energies. The shapes of the cross section curves shown in Fig. 4 vary for different products, particularly at low impact energies. Whereas the curves for $\mathrm{H}_{2} \mathrm{O}^{+}$ and $\mathrm{OH}^{+}$production are fairly flat, those for $\mathrm{H}^{+}, \mathrm{O}^{+}$, and $\mathrm{O}^{2+}$ exhibit increasingly pronounced maxima. The maximum partial cross section occurs at $70 \mathrm{keV}$ for $\mathrm{H}_{2} \mathrm{O}^{+}, 78 \mathrm{keV}$ for $\mathrm{OH}^{+}, 74 \mathrm{keV}$ for $\mathrm{H}^{+}, 65 \mathrm{keV}$ for $\mathrm{O}^{+}$, and $38 \mathrm{keV}$ for $\mathrm{O}^{2+}$. It

TABLE II. Values for the parameters in Eq. (1) derived by fitting the partial cross sections in Fig. 5 with the help of Eq. (1) originally derived by fitting the total cross section curve [17].

\begin{tabular}{crrrc}
\hline \hline Product ion & \multicolumn{1}{c}{$\mathrm{A}$} & \multicolumn{1}{c}{$\mathrm{B}$} & $\mathrm{C}$ & $\mathrm{D}$ \\
\hline $\mathrm{H}_{2} \mathrm{O}^{+}$ & 0.74 & 4.42 & 0.74 & 0.57 \\
$\mathrm{OH}^{+}$ & 1.29 & -0.53 & 0.52 & 0.65 \\
$\mathrm{O}^{+}$ & -0.34 & 1.25 & 0.08 & 0.63 \\
$\mathrm{O}^{2+}$ & -0.01 & 0.03 & 0.01 & 2.19 \\
$\mathrm{H}^{+}$ & -0.95 & 4.33 & 0.27 & 0.76 \\
\hline \hline
\end{tabular}

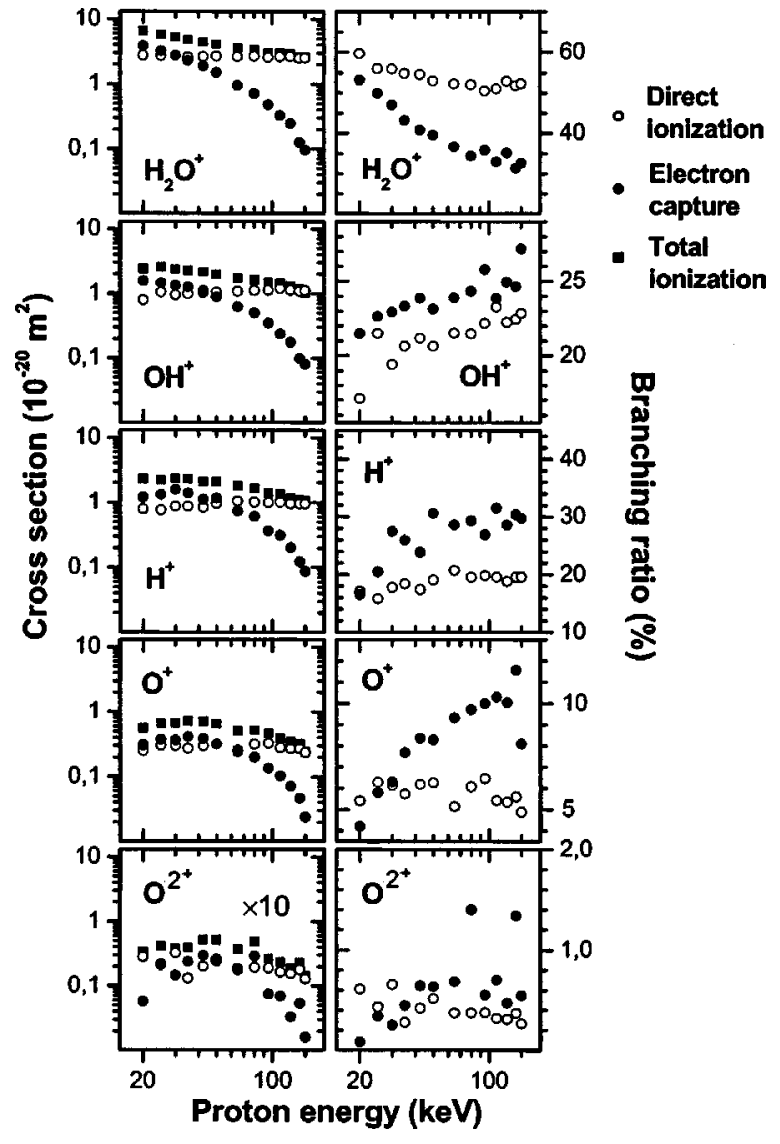

FIG. 5. Left side plots: absolute partial cross sections for proton impact ionization of $\mathrm{H}_{2} \mathrm{O}$. Right side plots: branching ratios (product cross sections divided by total ionization cross sections) for proton impact ionization of $\mathrm{H}_{2} \mathrm{O}$.

should be noted that, at low collision energies, $\mathrm{O}^{2+}$ formed by electron capture also contributes to Fig. 4 . In this case, the projectile is neutralized and an electron emitted simultaneously. Thus knowledge of the final charge state of the projectile is important to elucidate the kinetics of this collision process. For the other fragment ions, the contribution due to such electron capture processes is found to be negligible.

In the present experiment it is possible to distinguish between direct ionization (charge state of the projectile remains unchanged) and electron capture (neutralization of the projectile) for each partial cross section. This information can be used to derive the corresponding cross sections for direct ionization and for electron capture, separately. Figure 5 shows the partial cross sections and Fig. 6 the total cross sections. The two sets of cross sections are defined as differential in respect to the projectile charge state after the ionizing collision and the curves corresponding to direct ionization and to electron capture exhibit contrasting shapes. Direct ionization (open circles in Figs. 5 and 6) becomes the dominant process at energies above $60 \mathrm{keV}$, although the direct ionization cross sections begin to fall at higher energies. The partial cross sections for the production of $\mathrm{H}^{+}, \mathrm{O}^{+}$, and $\mathrm{O}^{2+}$ by electron capture show maxima at $30-40 \mathrm{keV}$. Conversely, the curves corresponding to the partial cross sections for $\mathrm{H}_{2} \mathrm{O}^{+}, \mathrm{OH}^{+}$, and the total cross section for ionization by electron capture show no such peaks in the present energy range. 


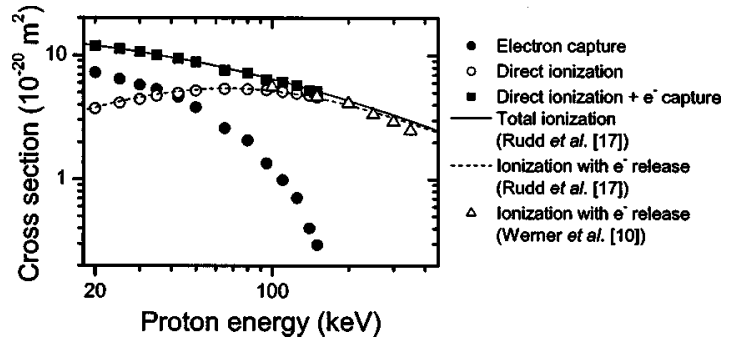

FIG. 6. Comparison between total ionization cross sections and cross sections direct ionization and electron capture for proton impact upon $\mathrm{H}_{2} \mathrm{O}$.

For all product ions, the cross section for production by electron capture decreases sharply with increasing energy, i.e., by two orders of magnitude from $20(-40) \mathrm{keV}$ to $150 \mathrm{keV}$.

The dashed line in Fig. 6 represents the cross section, $\sigma_{-}$, calculated using Eq. (1) and the solid line represents the total cross section for positive ion formation, $\sigma_{+}$, as given by the following equation [17]:

$$
\sigma_{+}\left(E_{p}\right)=\frac{4 \pi a_{0}^{2}}{\frac{x}{A \ln (1+x)+B}+\frac{1}{C x^{D}+F}},
$$

where $E_{p}$ is the proton energy in $\mathrm{eV}, x=\left(E_{p} / 1836\right) / 13.6$, and $a_{0}$ is the Bohr radius. The five parameters, $A=2.89, B$ $=4.42, C=1.48, D=0.75$, and $F=4.8$, were determined by fitting Eq. (2) to the experimental data of Rudd et al. [17]. At low proton energies the present cross sections for total ion production (solid squares) deviate slightly from the results of the equation. The open triangles correspond to the total cross sections published by Werner et al. [11] for positive ion formation with the release of at least one electron.

Although in the present experiment the charge state of the projectile when it hits the corresponding channeltron detector can be determined with precision, there are two possibilities that may lead to misinterpretation of a collision process.

(i) Protons that are neutralized before the interaction region can also initiate ionization events. Direct ionization of $\mathrm{H}_{2} \mathrm{O}$ by such a neutral $\mathrm{H}$ atom would thus be interpreted as an electron capture process. Therefore the proton beam is checked before each measurement for the presence of neutral $\mathrm{H}$ atoms. The ratio of protons to $\mathrm{H}$ atoms is measured without the water beam. For high collision energies this ratio is better than 10000:1, while at very low proton energies it decreases to $1000: 1$.

(ii) Lorentz field ionization of highly excited hydrogen Rydberg atoms. Electron capture from an ion often leads to the production of electronically highly excited atoms [27]. It is known that field ionization can occur also in the presence of a permanent magnetic field [28]. Thus production of such a Rydberg atom in an electron capture process and subsequent field ionization would be interpreted as a direct ionization event. Since the cross section for electron capture increases by more than 2 orders of magnitude when the projectile energy falls from $150 \mathrm{keV}$ to $30 \mathrm{keV}$, it is clear that field ionization of highly excited $\mathrm{H}^{*}$ has a greater effect on the data at low energies. However, previous $7-60 \mathrm{keV}$ proton

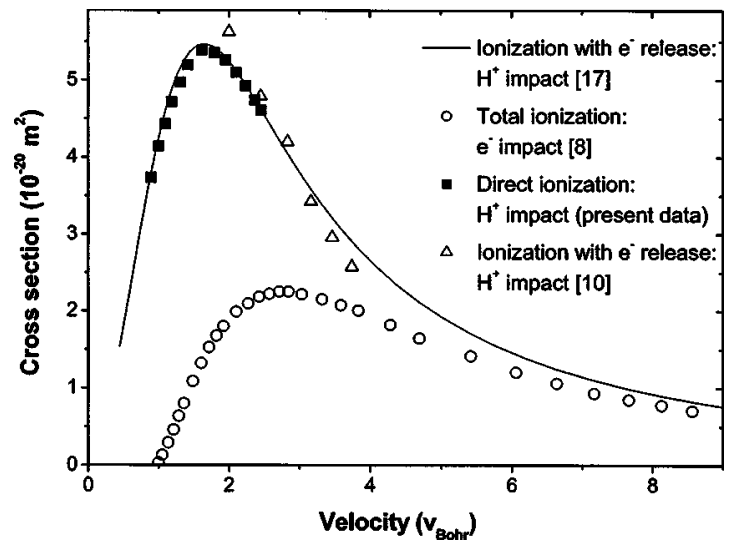

FIG. 7. Comparison between the total cross sections for ionization of $\mathrm{H}_{2} \mathrm{O}$ by electron impact reported by Straub et al. [8] and the total cross sections for direct ionization by proton impact (present data) and target ionization with electron release (Werner et al. [10]). The continuous line Eq. (1) corresponds to $\sigma_{-}$as defined by Eq. (1) of Rudd et al. [17].

impact experiments upon $\mathrm{H}$ and $\mathrm{Ar}$ showed the fraction of electron transfer collisions producing highly excited states of the target molecule to have a maximum value of about $0.3 \%$ [29] close to $30 \mathrm{keV}$. By analogy, the contribution of field ionization to the present data is assumed to be negligible.

In addition to partial differential cross sections, Fig. 5 also shows the branching ratios for direct ionization and electron capture. These values (plotted on the right) were derived by dividing the partial differential cross sections (plotted on the left) by the corresponding total differential cross sections shown in Fig. 6. The branching ratios for the individual product ions provide further insight into the mechanism for the fragmentation of water molecules upon proton impact. Over the full energy range studied, the branching ratio for $\mathrm{H}_{2} \mathrm{O}^{+}$ via direct ionization is greater than via electron capture. However, for all fragment ions, the branching ratios for direct ionization are smaller than for electron capture. This is in agreement with the observation that the relative intensity of fragment ions in the mass spectra is higher for the electron capture mechanism (see Fig. 2). Moreover, the branching ratio for the production of $\mathrm{H}_{2} \mathrm{O}^{+}$decreases with increasing collision energy for both channels, whereas for the production of fragment ions both branching ratios increase for greater collision energies.

For electron impact ionization the process of electron capture by the projectile is not possible. However, direct ionization by fast protons and electron impact ionization can be expected to be similar. Although the polarities are opposite, the instantaneous Coulomb interactions of both projectiles with the target molecule are equal. However, since the mass of a proton is a factor of 1836 larger than the mass of an electron, the interaction time (which is the important parameter for the inelastic interaction) between the two projectiles and a target molecule will be very different for the same collision energy. The velocity of a projectile is proportional to the interaction time. Figure 7 thus shows the present proton impact total cross sections for direct ionization $\left(\sigma_{\mathrm{di}}\right.$, solid squares) and the total electron impact ionization cross sec- 
tions reported by Straub et al. [8] (open circles) as a function of the projectile velocity. The open triangles correspond to the total cross sections for proton impact ionization with the release of at least one electron $\left(\sigma_{-}\right)$measured by Werner $e t$ al. [10]. The cross sections of Werner et al. will thus be slightly larger than those for direct ionization. However, at electron energies larger than $100 \mathrm{keV}$ the difference between $\sigma_{-}$and $\sigma_{\mathrm{di}}$ can be estimated to be less than $1 \%$ since electron capture becomes very unlikely (see Fig. 6). The solid line represents $\sigma_{-}$as calculated using Eq. (1) [17]. The velocity is given in multiples of the Bohr velocity $\left(v_{\text {Bohr }}=2.19\right.$ $\left.\times 10^{6} \mathrm{~ms}^{-1}\right)$. At high projectile velocities $\left(>5 v_{\text {Bohr }}\right)$ the cross section for electron and proton impact ionization are almost the same, whereas at lower velocities the cross sections for proton impact ionization are much larger. This result is similar to previous observations for the electron and proton impact ionization of the rare gases ([30] and references therein). Paludan et al. [30] reported that the single ionization cross sections (the major channel for the rare gases; the maximum contribution of double ionization is $\sim 5 \%$ of the single ionization channel) for $e^{-}, e^{+}, p^{+}$, and $p^{-}$ impact are identical for high velocity collisions (projectile velocity $\gg$ velocity of the bound target electrons as described by the first Born approximation). At lower velocities, however, the positive projectiles were observed to correspond to higher direct single ionization cross sections than the negatively charged particles. Paludan et al. [30] attributed this result to target polarization effects. They also commented that, at a fixed velocity, the lower kinetic energy of lighter projectiles reduces their cross sections relative to more massive counterparts as incident energy approaches the ionization potential of the target.

The comparison between the present partial cross sections for direct ionization by proton impact and those reported for electron impact by Straub et al. [8] can be extended. Straub et al. were unable to clearly separate the $\mathrm{H}_{2} \mathrm{O}^{+}$products from $\mathrm{OH}^{+}$and $\mathrm{O}^{+}$fragment ions. However, in addition to $\mathrm{H}_{2} \mathrm{O}$ they measured $\mathrm{D}_{2} \mathrm{O}$ ionization and observed the corresponding absolute partial cross sections to be equal within the experimental uncertainties (except for the $\mathrm{H}_{2}^{+}$and $\mathrm{D}_{2}{ }^{+}$), in agreement with earlier studies [31,32]. The mass resolution of the instrument used by Straub et al. was sufficiently high to measure partial cross sections for $\mathrm{O}^{+}, \mathrm{OD}^{+}$, and $\mathrm{D}_{2} \mathrm{O}^{+}$. Thus, for the heavy product ions, the electron impact ionization cross sections of $\mathrm{D}_{2} \mathrm{O}$ are available and can be compared with the present proton impact data for $\mathrm{H}_{2} \mathrm{O}$. The filled symbols in Fig. 8 represent the partial cross sections for direct ionization upon proton impact; big symbols correspond to the present data and small symbols to that of Werner et al. [10]. The partial electron impact ionization cross sections of Straub et al. [8] are represented by open symbols. The figure shows that the relative ordering of the partial cross sections is the same for both projectiles. However, as for the total single ionization cross sections (see above), at lower projectile velocities the partial cross sections are clearly greater for proton impact than for electron impact. Furthermore, the maximum cross sections occur at lower projectile velocities in the case of proton impact. This effect is more pronounced for the fragments with low absolute cross sections. In particular, whereas a proton with a velocity of twice the Bohr

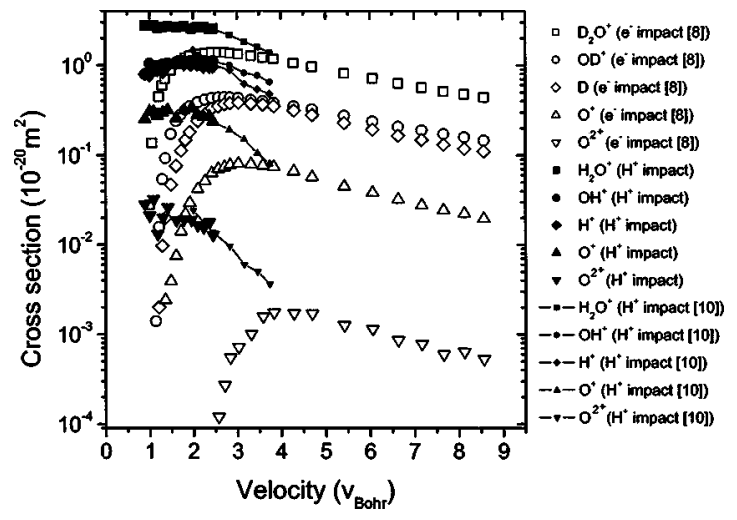

FIG. 8. Comparison between the electron impact partial ionization cross sections for $\mathrm{D}_{2} \mathrm{O}$ reported by Straub et al. [8] (open symbols) and the proton impact partial direct ionization cross sections for $\mathrm{H}_{2} \mathrm{O}$ measured in the present work (filled large symbols) and by Werner et al. [10] (filled small symbols with trend lines).

velocity leads to the maximum production of $\mathrm{O}^{2+}$, an electron with the same velocity does not have sufficient energy to produce this fragment ion at all. A similar shift of maximum cross section to lower projectile velocity for proton impact as opposed to electron impact was observed for the single and double partial ionization cross sections of the rare gases [30]. However, as in the case of the total cross sections shown in Fig. 7, the proton impact data match the electron impact data more closely at higher projectile velocities. The maximum partial cross sections for direct ionization by proton impact and by electron impact [8] are listed in Table III. Also given in the table are the thresholds for ion production from neutral $\mathrm{H}_{2} \mathrm{O}$ [33] expressed as energies and as velocities for proton and electron impact.

The final point of discussion relates to the presence of $\mathrm{H}_{2}{ }^{+}$ in the ionization mass spectra for proton impact upon $\mathrm{H}_{2} \mathrm{O}$. As the $\mathrm{H}_{2}{ }^{+}$ion signal is strongly correlated with the density

TABLE III. The values in the column designated Max $\sigma_{P I}$ are the maximum values of the partial cross sections for direct ionization upon proton impact. The column designated Max $\sigma_{E I}$ contains the maximum values of the partial cross sections for electron impact ionization. AE stands for appearance energy, meaning the threshold energy needed to produce a particular product ion from neutral water. Except for the $\mathrm{O}^{2+}$, the values are taken from the NIST tables [33]. The appearance energy for doubly charged oxygen was determined by linear extrapolation of the threshold region of the cross section values given by Straub et al. [8]. The appearance energies are converted into proton and electron impact velocities in the double column labeled Av.

\begin{tabular}{ccccccc}
\hline \hline & & & & \multicolumn{2}{c}{$\operatorname{Av}\left(v_{\text {Bohr }}\right)$} \\
\cline { 5 - 6 } $\begin{array}{c}\text { Product } \\
\text { ion }\end{array}$ & $\begin{array}{c}\text { Max } \sigma_{P I} \\
\left(10^{-20} \mathrm{~m}^{2}\right)\end{array}$ & $\begin{array}{c}\text { Max } \sigma_{E I} \\
\left(10^{-20} \mathrm{~m}^{2}\right)\end{array}$ & $\begin{array}{c}\mathrm{AE} \\
(\mathrm{eV})\end{array}$ & $\mathrm{H}^{+}$ & $e^{-}$ \\
\hline $\mathrm{H}_{2} \mathrm{O}^{+}$ & 2.713 & 1.38 & 12.65 & 0.022 & 0.96 \\
$\mathrm{OH}^{+}$ & 1.208 & 0.441 & 18.08 & 0.026 & 1.15 \\
$\mathrm{O}^{+}$ & 0.338 & 0.0802 & 26.8 & 0.033 & 1.40 \\
$\mathrm{O}^{2+}$ & 0.0217 & 0.00175 & 86.3 & 0.059 & 2.51 \\
$\mathrm{H}^{+}$ & 1.263 & 0.382 & 18.2 & 0.027 & 1.16 \\
\hline \hline
\end{tabular}


of water beam, this peak cannot be attributed to neutral hydrogen molecules from the ion source. The complementary fragment to $\mathrm{H}_{2}^{+}$production ion is a neutral oxygen atom. Due to momentum conservation, the $\mathrm{H}_{2}^{+}$will carry away almost $90 \%$ of the kinetic energy released in such a dissociation event. Therefore, one can expect the counting losses due to discrimination for this light fragment ion to be significant. Table I indicates that the discrimination factor for $\mathrm{H}_{2}{ }^{+}$ will be larger than 2 . The two mass spectra shown in Fig. 2 (both averaged over all projectile energies in the regions corresponding to of $\mathrm{H}_{2}^{+}$and $\mathrm{O}^{2+}$ ) show the cross sections for the production of $\mathrm{O}^{2+}$ and $\mathrm{H}_{2}^{+}$to be of the same order of magnitude. This is in good agreement with the results of Straub et al. [8] for electron impact ionization (see also Fig. 8).

\section{CONCLUSIONS}

The present work provides a complete set of partial and total cross sections for the ionization of water molecules by proton impact in the energy range $20-150 \mathrm{keV}$. The resultant ions are mass analyzed in coincidence with the determination of the projectile charge state postinteraction. Thus direct ionization can be distinguished from electron capture events. In order to achieve absolute cross sections, the present results are calibrated using the cross sections for positive ion pro- duction and electron emission by $\mathrm{H}_{2} \mathrm{O}$ upon proton impact measured by Rudd et al. [17]. Corrections for discrimination effects associated with kinetic energy release during fragmentation are made by comparison with the data of Werner et al. [10].

No evidence is observed for the production of negatively charged ion products or for ionization by double electron capture. $\mathrm{H}_{2}^{+}$fragments are detected for the first time in proton- $\mathrm{H}_{2} \mathrm{O}$ collisions. For all product ions, direct ionization is found to be the dominant process at high energies. As a proportion of $\mathrm{H}_{2} \mathrm{O}^{+}$formation, electron capture produces higher yields of fragment ions. Comparisons are made between the present cross sections for direct ionization of water proton impact and the electron impact ionization data reported by Straub et al. [8]. At high projectile velocities the cross sections for electron and proton impact ionization are similar, whereas at lower velocities the cross sections for proton impact ionization are found to be significantly greater.

\section{ACKNOWLEDGMENTS}

This work was supported by the FWF and the ÖNB, Wein, Austria, by the French and Austrian governments through the Amadee program, by the European Commission, Brussels, and by the French and Morocco governments through the Convention CNRST/CNRS.
[1] E. W. McDaniel, J. B. A. Mitchell, and M. E. Rudd, Atomic Collisions (Wiley, New York, 1993).

[2] C. S. Enos, A. R. Lee, and A. G. Brenton, Int. J. Mass Spectrom. Ion Processes 104, 137 (1991).

[3] D. H. Katayama, R. E. Huffman, and C. L. O'Bryan, J. Chem. Phys. 59, 4309 (1973).

[4] K. Mitsuke, T. Takami, and K. Ohno, J. Chem. Phys. 91, 1618 (1989).

[5] G. H. Olivera, C. Caraby, P. Jardin, A. Cassimi, L. Adoui, and B. Gervais, Phys. Med. Biol. 43, 2347 (1998).

[6] M. Dingfelder, M. Inokuti, and H. G. Paretzke, Radiat. Phys. Chem. 59, 255 (2000).

[7] B. Boudaiffa, P. Cloutier, D. Hunting, M. A. Hues, and L. Sanche, Science 287, 1658 (2000).

[8] H. C. Straub, B. G. Lindsay, K. A. Smith, and R. F. Stebbings, J. Chem. Phys. 108, 109 (1998).

[9] O. Sueoka, S. Mori, and K. Katayama, J. Phys. B 20, 3237 (1987).

[10] U. Werner, K. Beckord, J. Becker, and H. O. Lutz, Phys. Rev. Lett. 74, 1962 (1995).

[11] F. Gobet, B. Farizon, M. Farizon, M. J. Gaillard, M. Carre, M. Lezius, P. Scheier, and T. D. Märk, Phys. Rev. Lett. 86, 3751 (2001).

[12] V. Tarnovsky, H. Deutsch, and K. Becker, J. Chem. Phys. 109, 932 (1998).

[13] D. W. Koopman, Phys. Rev. 166, 57 (1968).

[14] L. H. Toburen, M. Y. Nakai, and R. A. Langley, Phys. Rev. 171, 114 (1968).

[15] L. H. Toburen and W. E. Wilson, J. Chem. Phys. 66, 5202
(1977).

[16] L. H. Toburen, W. E. Wilson, and R. J. Popowich, Radiat. Res. 82, 27 (1980).

[17] M. E. Rudd, T. V. Goffe, R. D. DuBois, and L. H. Toburen, Phys. Rev. A 31, 492 (1985).

[18] M. E. Rudd, Akio Itoh, and T. V. Goffe, Phys. Rev. A 32, 2499 (1985).

[19] M. A. Bolorizadeh and M. E. Rudd, Phys. Rev. A 33, 882 (1986).

[20] M. A. Bolorizadeh and M. E. Rudd, Phys. Rev. A 33, 888 (1986).

[21] M. A. Bolorizadeh and M. E. Rudd, Phys. Rev. A 33, 893 (1986).

[22] M. Carré, M. Druetta, M. L. Gaillard, H. H. Bukow, M. Horani, A. L. Roche, and M. Velghe, Mol. Phys. 40, 1453 (1980).

[23] W. C. Wiley and I. H. McLaren, Rev. Sci. Instrum. 16, 1150 (1955).

[24] T. J. Morgan, K. H. Berkner, W. G. Graham, R. V. Pyle, and J. W. Stearns, Phys. Rev. A 14, 664 (1976).

[25] H. U. Poll, C. Winkler, D. Margreiter, V. Grill, and T. D. Märk, Int. J. Mass Spectrom. Ion Processes 112, 1 (1992).

[26] P. Scheier, G. Senn, S. Matt, and T. D. Märk, Int. J. Mass Spectrom. Ion Processes 172, L1 (1998).

[27] S. A. Aseyev, Y. A. Kudryavtsev, and V. V. Petrunin, J. Phys. B 24, L647 (1991).

[28] S. Borneis, F. Bosch, T. Engel, M. Jung, I. Klaft, O. Klepper, T. Kühl, D. Marx, R. Moshammer, R. Neumann, S. Schröder, P. Seelig, and L. Völker, Phys. Rev. Lett. 72, 207 (1994). 
[29] J. E. Bayfield, G. A. Khayrallah, and P. M. Koch, Phys. Rev. A 9, 209 (1974).

[30] K. Paludan, G. Laricchia, P. Ashley, V. Kara, J. Moxom, H. Bluhme, H. Knudsen, U. Mikkelsen, S. P. Møller, E. Uggerhøj, and E. Morenzoni, J. Phys. B 30, L581 (1997).
[31] N. L. Djuric, I. M. Cadez, and M. V. Kurepa, Int. J. Mass Spectrom. Ion Processes 83, R7 (1988).

[32] T. D. Märk and F. Egger, Int. J. Mass Spectrom. Ion Phys. 20, 89 (1976).

[33] NIST table. 